\title{
$T=0$ Partition Functions for Potts Antiferromagnets on Square Lattice Strips with (Twisted) Periodic Boundary Conditions
}

\author{
Norman Biggs® \\ Centre for Discrete and Applicable Mathematics \\ London School of Economics \\ London WC2A $2 \mathrm{AE}$ \\ $\mathrm{UK}$ \\ Robert Shrock* \\ Institute for Theoretical Physics \\ State University of New York \\ Stony Brook, N. Y. 11794-3840 \\ USA
}

\begin{abstract}
We present exact calculations of the zero-temperature partition function for the $q$-state Potts antiferromagnet (equivalently, the chromatic polynomial) for two families of arbitrarily long strip graphs of the square lattice with periodic boundary conditions in the transverse direction and (i) periodic (ii) twisted periodic boundary conditions in the longitudinal direction, so that the strip graphs are embedded on a (i) torus (ii) Klein bottle. In the limit of infinite length, we calculate the exponent of the entropy, $W(q)$, show it to be the same for (i) and (ii), and determine its analytic structure.
\end{abstract}

*email: n.l.biggs@lse.ac.uk

**email: robert.shrock@sunysb.edu 
The chromatic polynomial $P(G, q)$ counts the number of ways that one can color a graph $G$ with $q$ colors such that no two adjacent vertices have the same color [1] (for reviews, see [2]-[泪). The least positive integer $q$ for which $P(G, q)$ is nonzero is the chromatic number, $\chi(G)$. Besides its intrinsic mathematical interest, the chromatic polynomial has an important connection with statistical mechanics since it is the zero-temperature partition function of the $q$-state Potts antiferromagnet (AF) [5, G] on $G: P(G, q)=Z(G, q, T=0)_{P A F}$. The Potts AF exhibits nonzero ground-state entropy $S_{0} \neq 0$ (without frustration) for sufficiently large $q$ on a given lattice graph and is thus an exception to the third law of thermodynamics. This is equivalent to a ground state degeneracy per site $W>1$, since $S_{0}=k_{B} \ln W$. Denoting the number of vertices of $G$ as $n=v(G)$ and assuming a reasonable definition of $\{G\}=$ $\lim _{n \rightarrow \infty} G$, we have $W(\{G\}, q)=\lim _{n \rightarrow \infty} P(G, q)^{1 / n}$. Since $P(G, q)$ is a polynomial, one can generalize $q$ from $\mathbb{Z}_{+}$to $\mathbb{C}$. The zeros of $P(G, q)$ in the complex $q$ plane are called chromatic zeros; a subset of these may form an accumulation set in the $n \rightarrow \infty$ limit, denoted $\mathcal{B}$, which is the continuous locus of points where $W(\{G\}, q)$ is nonanalytic [ $\mathbb{8}$, 16, ] ( $\mathcal{B}$ may be null, and $W$ may also be nonanalytic at certain discrete points). The maximal region in the complex $q$ plane to which one can analytically continue the function $W(\{G\}, q)$ from physical values where there is nonzero ground state entropy is denoted $R_{1}$. The maximal value of $q$ where $\mathcal{B}$ intersects the (positive) real axis is labelled $q_{c}(\{G\})$.

We consider strips of the square lattice with arbitrary length $L_{x}=m$ vertices and fixed width $L_{y}$ vertices (with the longitudinal and transverse directions taken to be $\hat{x}$ and $\hat{y}$ ). The chromatic polynomials for the cyclic and Möbius strip graphs of the square lattice were calculated for $L_{y}=2$ in [9] (see also [10]-[12]) and for $L_{y}=3$ in [13, 14]. After studies of the chromatic zeros for $L_{y}=2$ in [9, 15, 16], $W$ and $\mathcal{B}$ were determined for this case in [7] and for $L_{y}=3$ in [13]. An important question concerns the effect of boundary conditions (BC's), and hence graph topology, on $P, W$, and $\mathcal{B}$. We use the symbols $\mathrm{FBC}_{y}$ and $\mathrm{PBC}_{y}$ for free and periodic transverse boundary conditions and $\mathrm{FBC}_{x}, \mathrm{PBC}_{x}$, and $\mathrm{TPBC}_{x}$ for free, periodic, and twisted periodic longitudinal boundary conditions. The term "twisted" means that the longitudinal ends of the strip are identified with reversed orientation. These strip graphs can be embedded on surfaces with the following topologies:U : (i) $\left(\mathrm{FBC}_{y}, \mathrm{FBC}_{x}\right)$ : strip; (ii) $\left(\mathrm{PBC}_{y}, \mathrm{FBC}_{x}\right)$ : cylindrical; (iii) $\left(\mathrm{FBC}_{y}, \mathrm{PBC}_{x}\right)$ : cylindrical (denoted cyclic here); (iv)

\footnotetext{
${ }^{1}$ At certain special points $q_{s}$ (typically $q_{s}=0,1, . ., \chi(G)$ ), one has the noncommutativity of limits $\lim _{q \rightarrow q_{s}} \lim _{n \rightarrow \infty} P(G, q)^{1 / n} \neq \lim _{n \rightarrow \infty} \lim _{q \rightarrow q_{s}} P(G, q)^{1 / n}$, and hence it is necessary to specify the order of the limits in the definition of $W\left(\{G\}, q_{s}\right)[7]$. We use the first order of limits here; this has the advantage of removing certain isolated discontinuities in $W$.

${ }^{2}$ For the triangular lattice with cylindrical BC's, $W$ and $\mathcal{B}$ were calculated in 17 . Other calculations of $P, W$, and $\mathcal{B}$ have been performed for strips having BC's of type (i) [18]-20], (ii) 22], (iii) [21]-22], (iv) [21, 14].
} 
$\left(\mathrm{FBC}_{y}, \mathrm{TPBC}_{x}\right)$ : Möbius; (v) $\left(\mathrm{PBC}_{y}, \mathrm{PBC}_{x}\right)$ : torus; and (vi) $\left(\mathrm{PBC}_{y}, \mathrm{TPBC}_{x}\right)$ : Klein bottle. Here we present and analyze chromatic polynomials for the strip graph of the square lattice with $L_{y}=3$ (i.e. cross sections forming triangles) and boundary conditions of type (v) and (vi): torus and Klein bottle. We recall that unlike graphs of type (i)-(v), the Klein bottle graph (vi) cannot be embedded without self-intersection in $\mathbb{R}^{3}$. For $L_{x}=m \geq 2$ where they are well defined, the $L_{y}=3$ torus and Klein bottle graphs have $n=L_{x} L_{y}$ vertices, $e=2 n$ edges, the same girth $g$ (length of minimum closed circuit) and number $k_{g}$ of circuits of length $g$, and the respective chromatic numbers $\chi=3$ and $\chi=4$.

We label a particular type of strip graph as $G_{s}$ and the specific graph of length $L_{x}=m$ repeated subgraph units, e.g. columns of squares in the case of the square strip, as $\left(G_{s}\right)_{m}$. If one thinks of the graph as embedded on a rectangular strip of paper, with its upper and lower sides glued together and its longitudinal ends glued with direct or reversed orientation, then $L_{x}$ is the length of this strip of paper in subgraph units. Writing

$$
P\left(\left(G_{s}\right)_{m}, q\right)=\sum_{j=0}^{n-1}(-1)^{j} h_{n-j} q^{n-j}
$$

and using the results that 25, 3] $h_{n-j}=\left(\begin{array}{l}e \\ j\end{array}\right)$ for $0 \leq j<g-1\left(\right.$ whence $h_{n}=1$ and $h_{n-1}=e$ ) and $h_{n-(g-1)}=\left(\begin{array}{c}e \\ g-1\end{array}\right)-k_{g}$, it follows that for $m$ greater than the above-mentioned minimal value, these $h_{j}$ 's are the same for the torus and Klein bottle of each type $G_{s}$. For a given $G_{s}$, as $m$ increases, the $h_{n-j}$ 's for the torus and Klein bottle graphs become equal for larger $j$.

A generic form for chromatic polynomials for recursively defined families of graphs, of which strip graphs $G_{s}$ are special cases, is

$$
P\left(\left(G_{s}\right)_{m}, q\right)=\sum_{j=1}^{N_{\lambda}} c_{j}(q)\left(\lambda_{j}(q)\right)^{m}
$$

where $c_{j}(q)$ and the $N_{\lambda}$ terms $\lambda_{j}(q)$ depend on the type of strip graph $G_{s}$ but are independent of $m$.

For an $L_{y}=3, L_{x}=m$ strip with $\left(\mathrm{PBC}_{y}, \mathrm{FBC}_{x}\right)$ one has [20] $P\left(s q\left(L_{y}=3\right)_{m}, P B C_{y}, F B C_{x}, q\right)=$ $q(q-1)(q-2)\left(q^{3}-6 q^{2}+14 q-13\right)^{m-1}$, whence

$$
W\left(s q\left(L_{y}=3\right), P B C_{y}, F B C_{x}, q\right)=\left(q^{3}-6 q^{2}+14 q-13\right)^{1 / 3}
$$

with $\mathcal{B}=\emptyset$.

These BC's can all be implemented in a manner that is uniform in the length $L_{x}$; the case (vii) $\left(\mathrm{TPBC}_{y}, \mathrm{TPBC}_{x}\right)$ with the topology of the projective plane requires different identifications as $L_{x}$ varies and will not be considered here. For connections between topology and graph properties, see e.g. 223, 24. 
In order to calculate $P$, one may use recursive methods based on iterative use of deletioncontraction theorems [9, 10, 18] or a coloring compatibility matrix method described in [12, 26]. For the $L_{y}=3$ torus $(t)$ graphs, one finds

$$
P\left(s q\left(L_{y}=3\right)_{m}, P B C_{y}, P B C_{x}, q\right)=\sum_{j=1}^{8} c_{t, j}\left(\lambda_{t, j}\right)^{m}
$$

where

$$
\begin{gathered}
\lambda_{t, 1}=-1, \quad c_{t, 1}=q^{3}-6 q^{2}+8 q-1, \\
\lambda_{t, 2}=q^{3}-6 q^{2}+14 q-13, \quad c_{t, 2}=1, \\
\lambda_{t, 3}=q-1, \quad c_{t, 3}=\frac{(q-1)(q-2)}{2}, \\
\lambda_{t, 4}=q-4, \quad c_{t, 4}=(q-1)(q-2), \\
\lambda_{t, 5}=q-2, \quad c_{t, 5}=q(q-3), \\
\lambda_{t, 6}=q-5, \quad c_{t, 6}=\frac{q(q-3)}{2}, \\
\lambda_{t, 7}=-\left(q^{2}-7 q+13\right), \quad c_{t, 7}=q-1, \\
\lambda_{t, 8}=-(q-2)^{2}, \quad c_{t, 8}=2(q-1) .
\end{gathered}
$$

For the $L_{y}=3$ Klein $(K)$ bottle graphs one finds

$$
P\left(s q\left(L_{y}=3\right)_{m}, P B C_{y}, T P B C_{x}, q\right)=\sum_{j=1}^{5} c_{K, j}\left(\lambda_{K, j}\right)^{m}
$$

where

$$
\begin{gathered}
\lambda_{K, 1}=\lambda_{t, 1}=-1, \quad c_{K, 1}=-(q-1), \\
\lambda_{K, 2}=\lambda_{t, 2}=q^{3}-6 q^{2}+14 q-13, \quad c_{K, 2}=c_{t, 2}=1, \\
\lambda_{K, 3}=\lambda_{t, 3}=q-1, \quad c_{K, 3}=-c_{t, 3}=-\frac{(q-1)(q-2)}{2}, \\
\lambda_{K, 4}=\lambda_{t, 6}=q-5, \quad c_{K, 4}=c_{t, 6}=\frac{q(q-3)}{2}, \\
\lambda_{K, 5}=\lambda_{t, 7}=-\left(q^{2}-7 q+13\right), \quad c_{K, 5}=c_{t, 7}=q-1 .
\end{gathered}
$$

The terms $\lambda_{t, j}, j=4,5,8$ do not enter in eq. (13). We contrast this with earlier findings. For a given strip, $N_{\lambda}$ was found to be larger for $\left(\mathrm{FBC}_{y}, \mathrm{PBC}_{x}\right)$ than $\left(\mathrm{FBC}_{y}, \mathrm{FBC}_{x}\right)$ [18, 21, 13]. For the $L_{y}=3$ square lattice strip case, $N_{\lambda}=2$ for $\left(\mathrm{FBC}_{y}, \mathrm{FBC}_{x}\right)$ but $N_{\lambda}=1$ for $\left(\mathrm{PBC}_{y}, \mathrm{FBC}_{x}\right)$, because of the special feature that the cross sections were complete graphs, $K_{p}$ with $p=3$ 


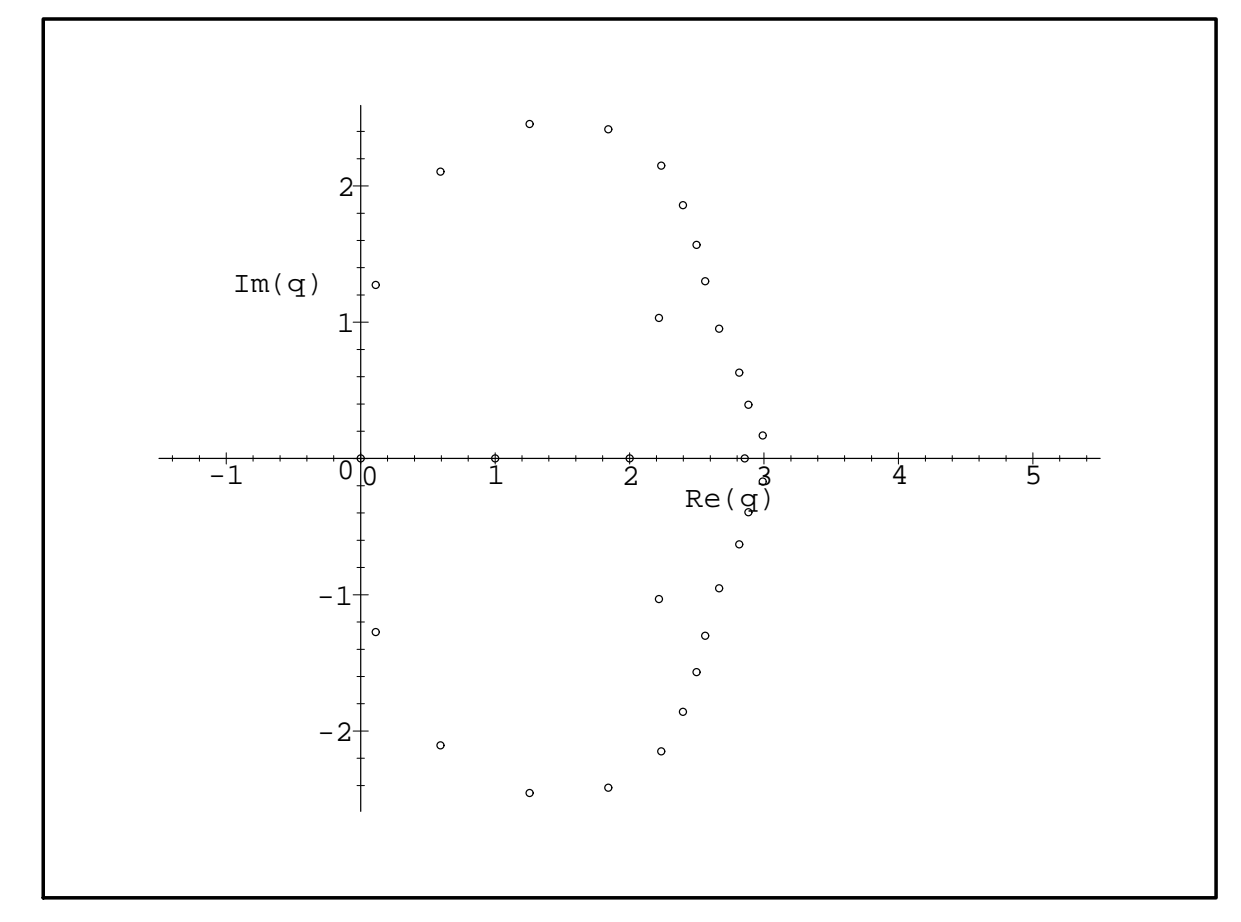

Figure 1: Chromatic zeros for the $L_{y}=3, L_{x}=m=10$ torus graph.

and hence the intersection theorem led to a factorized, monomial form for $P$. It was found 113, 14 that for a given type of lattice strip, $N_{\lambda}$ is the same for the $\left(\mathrm{FBC}_{y}, \mathrm{PBC}_{x}\right)=$ cyclic and $\left(\mathrm{FBC}_{y}, \mathrm{TPBC}_{x}\right)=$ Möbius topologies, although the $c_{j}$ 's were, in general, different. The present results show that reversal of orientation in the identification of opposite ends of a strip can lead to a change in $N_{\lambda}$. f

Let $C=\sum_{j=1}^{N_{\lambda}} c_{j}$. We find $C=P\left(K_{3}, q\right)=q(q-1)(q-2)$ for the $L_{y}=3$ torus graphs and $C=0$ for the $L_{y}=3$ Klein bottle graphs. The zero results from the special constraints introduced by the boundary conditions and is analogous to the fact that $C=0$ for the $L_{y}=2$ Möbius square strip [9] and its homeomorphic expansions [21]. However, not all Möbius strip graphs have $C=0$; for example, for the $L_{y}=3$ Möbius strips of the square and kagomé lattices, $C=q(q-1)$ and $C=q$, respectively [14].

Chromatic zeros for the $L_{y}=3, m=10$ torus graph are shown in Fig. 1; with this value of $m$, the chromatic zeros for the Klein bottle graph are quite similar. The locus $\mathcal{B}$ and the $W$ functions are the same for the torus and Klein bottle graph families. We find $q_{c}=3$, which, interestingly, is the same value as for the infinite $2 \mathrm{D}$ square lattice. The locus $\mathcal{B}$ has support for $R e(q) \geq 0$ and separates the $q$ plane into three regions. The outermost one, region $R_{1}$, extends to infinite $|q|$ and includes the intervals $q \geq 3$ and $q \leq 0$ on the real $q$ axis. Region

\footnotetext{
${ }^{4} \mathrm{~A}$ different sort of change in $P$, accompanied by a change in $\mathcal{B}$, can be obtained if one considers a homogeneous recursive family and the same family with a finite inhomogeneous subgraph inserted, e.g., the "rope ladder" graphs of [16] or two such subgraphs forming ends, viz., the $J\left(\prod H\right) I$ strip graphs in 18 .
} 
$R_{2}$ includes the real interval $2 \leq q \leq 3$ and extends upward and downward to the complex conjugate triple points on $\mathcal{B}$ at $q_{t}$ and $q_{t}^{*}$, where $q_{t} \simeq 2.5+1.4 i$. Region $R_{3}$ is the innermost one and includes the real interval $0 \leq q \leq 2$. The boundary between $R_{2}$ and $R_{3}$ curves to the right as one increases $|\operatorname{Im}(q)|$, extending from $q=2$ upward to $q_{t}$ and downward to $q_{t}^{*}$. As is evident in Fig. 1, the density of chromatic zeros on the $R_{1}-R_{3}$ boundary near $q=0$ and on the $R_{3}-R_{2}$ boundary is somewhat smaller than on the right-hand part of the $R_{2}-R_{1}$ boundary.

In region $R_{1}, \lambda_{t, 2}=\lambda_{K, 2}$ is the dominant $\lambda_{j}$, so

$$
W=\left(q^{3}-6 q^{2}+14 q-13\right)^{1 / 3}, \quad q \in R_{1} .
$$

The fact that this is the same as $W$ for the $\left(\mathrm{PBC}_{y}, \mathrm{FBC}_{x}\right)$ case, eq. (3), is a general result. The importance of the $\mathrm{PBC}_{y}$ is evident from the fact that for the same width of three squares, the strip with $\left(\mathrm{FBC}_{y}, \mathrm{FBC}_{x}\right)$ yields a different $W$ [18.

In region $R_{2} \lambda_{t, 6}=\lambda_{K, 4}$ is dominant, so

$$
|W|=|q-5|^{1 / 3}, \quad q \in R_{2}
$$

(in regions other than $R_{1}$, only $|W|$ can be determined unambiguously [7]). In region $R_{3}$, $\lambda_{t, 7}=\lambda_{K, 5}$ is dominant, so

$$
|W|=\left|q^{2}-7 q+13\right|^{1 / 3}, \quad q \in R_{3} .
$$

The outer boundary separating $R_{1}$ from the inner two regions is oblate, extending out to a maximum of about $|\operatorname{Im}(q)| \simeq 2.5$ for $\operatorname{Re}(q) \simeq 1.5$ (and passing through $q=0$ and 3 ). All the three points, $q=0,2,3$, where $\mathcal{B}$ crosses the real $q$ axis, it does so vertically. The present results are in accord with the inference [18, 13] that for a recursive graph with regular lattice structure, a necessary and sufficient condition for $\mathcal{B}$ to separate the $q$ plane into two or more regions is that it contains a global circuit, i.e. a path along a lattice direction whose length goes to infinity as $n \rightarrow \infty$; here this is equivalent to $\mathrm{PBC}_{x}$. The fact that $\mathcal{B}$ is the same for these torus and Klein families means that none of $\lambda_{t, j}, j=4,5,8$ is a dominant term.

Our calculations of the zero-temperature Potts antiferromagnet partition functions (chromatic polynomials) and exponential of the entropy, $W$, for $L_{y}=3$ strips of the square lattice with periodic transverse and periodic and twisted periodic longitudinal boundary conditions (torus and Klein bottle graphs) thus elucidates the role that these boundary conditions and the associated topologies play; the torus and Klein bottle graphs have interestingly different chromatic polynomials, with different $N_{\lambda}$, but the $W$ functions and hence the boundaries $\mathcal{B}$ are the same.

The research of R. S. was supported in part by the U. S. NSF grant PHY-97-22101. 


\section{References}

[1] Birkhoff, G. D. 1912 Ann. of Math. 14, 42.

[2] Read, R. C. 1968 J. Combin. Theory 4, 52.

[3] Read, R. C. and Tutte, W. T. 1988 "Chromatic Polynomials", in Selected Topics in Graph Theory, 3, (Academic Press, New York), p. 15.

[4] Biggs, N. L. 1993 Algebraic Graph Theory (Cambridge Univ. Press, Cambridge).

[5] Potts, R. B. 1952 Proc. Camb. Phil. Soc. 48, 106.

[6] Wu, F. Y. 1982 Rev. Mod. Phys. 54, 235.

[7] Shrock, R. and Tsai, S.-H. 1997 Phys. Rev. E55, 5165.

[8] Beraha, S., Kahane, J., and Weiss, N. 1980 J. Combin. Theory B 28, 52.

[9] Biggs, N. L., Damerell, R. M. and Sands, D. A. 1972 J. Combin. Theory B 12, 123.

[10] Biggs, N. L. and Meredith, G. H. 1976 J. Combin. Theory B 20, 5.

[11] Biggs, N. L. 1977 Bull. London Math. Soc. 9, 54.

[12] Biggs, N. L. LSE report LSE-CDAM-99-03 (May 1999), to appear.

[13] Shrock, R. and Tsai, S.-H. 1999 Phys. Rev. E, in press; Stony Brook report ITP-SB-9912 (April, 1999).

[14] Shrock, R., Stony Brook report ITP-SB-99-23 (June, 1999).

[15] Read, R. C. 1988 in Proc. 5th Caribbean Conf. on Combin. and Computing.

[16] Read, R. C. and Royle, G. F. 1991 in Graph Theory, Combinatorics, and Applications (Wiley, NY), vol. 2, p. 1009.

[17] Baxter, R. J. 1987 J. Phys. A 20, 5241.

[18] Roček, M., Shrock, R., and Tsai, S.-H. 1998 Physica A252, 505; ibid. A259, 367.

[19] Shrock, R. and Tsai, S.-H. 1998 Physica A259, 315.

[20] Shrock, R. and Tsai S.-H. 1998 Phys. Rev. E58, 4332, cond-mat/9808057. 
[21] Shrock, R. and Tsai, S.-H. 1999 J. Phys. A Lett. 32 L195.

[22] Shrock, R. and Tsai, S.-H. 1999 J. Phys. A, in press (cond-mat/9905431).

[23] Saaty, T. L. and Kainen, P. C. 1977 The Four-Color Problem (McGraw-Hill, New York), p. 45 .

[24] White, A. T. and Beineke, L. W. 1978 in Beineke, L. W. and Wilson, R. J. Selected Topics in Graph Theory (Academic, New York) p. 15.

[25] Meredith, G. H. 1972 J. Combin. Theory B 13, 14.

[26] Biggs, N. L. LSE report LSE-CDAM-99-05 (June 1999). 